Results Patients in the third HS-TnT tercile were 6 times more likely to sustain a cardiovascular event compared to those in the first tercile [unadjusted RR 6.11 (95\% CI 2.98 to 12.50)]. A high HS-TnT predicted adverse clinical outcomes independent of conventional risk factors, left ventricular dysfunction and left ventricular hypertrophy on echocardiography [adjusted RR 2.69 (95\% CI 1.45 to 5.00)]. Patients with persistent HS-TnT elevation at 7 weeks were also at an increased risk of cardiovascular events compared to those with an initial high HS-TnT which then normalised [unadjusted RR 3.39 (95\% CI 2.02 to 5.68$)$ ].

Conclusion We have demonstrated for the first time the prognostic utility of a single 7-week HS-TnT measurement in routine ACS patients and that it could be used to assist medium term risk stratification in this patient cohort. Future studies should evaluate whether tailoring specific treatment interventions to higher risk individuals as identified by an elevated HS-TnT during the convalescence phase of ACS would improve clinical outcomes.

\section{PRIOR CORONARY ARTERY BYPASS GRAFT PATIENTS TREATED WITH PRIMARY PERCUTANEOUS CORONARY INTERVENTION HAVE HIGHER LONG-TERM ADVERSE EVENT RATES}

doi:10.1136/heartjnl-2012-301877b.130

S M Hamshere,* D A Jones, M Akhtar, K S Rathod, A Graham, 0 Guttmann, S Gallagher, A Jain, C Knight, A Mathur, A Wragg. London Chest Hospital, London, UK

Background Limited information exists regarding procedural success and clinical outcomes of ST-segment elevation myocardial infarction (STEMI) patients with previous CABG undergoing primary PCI. We sought to compare outcomes in STEMI patients undergoing primary percutaneous coronary intervention (PCI) with or without previous coronary artery bypass grafts (CABG).

Methods Clinical information was analysed from a prospective database on 2322 STEMI patients who underwent Primary PCI between January 2004 and May 2010 at a London centre. 104 of 2322 (4.5\%) patients had prior CABG. MACE was defined as a composite of death, stroke and target vessel revascularisation (TVR). Outcome assessed by all-cause mortality information provided by the Office of National Statistics via the BCIS CCAD national audit.

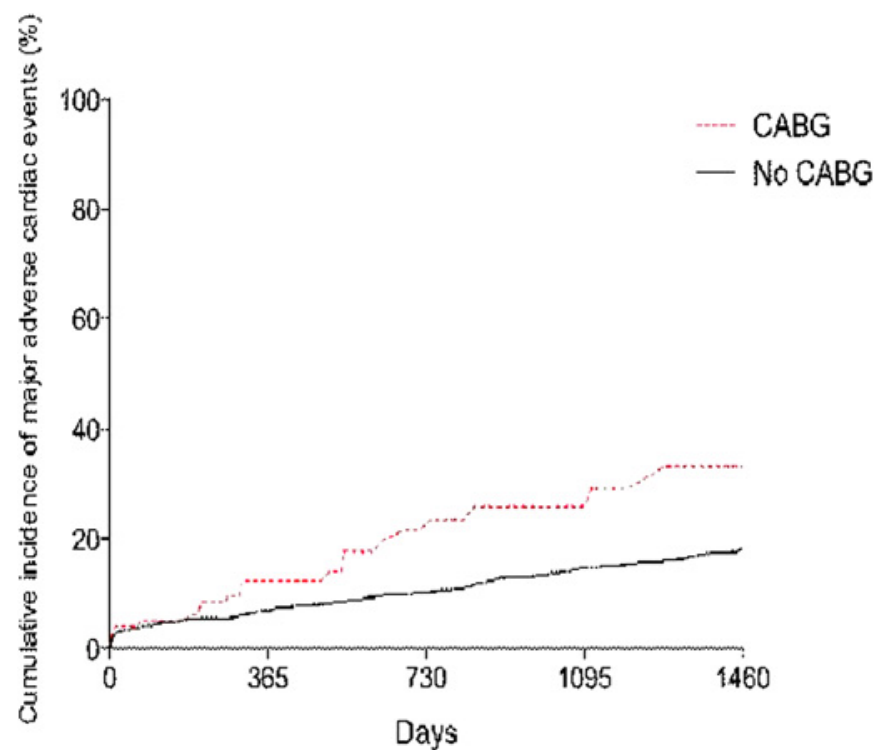

Abstract 130 Figure 1
Results Patients with previous CABG were older, had more associated comorbidity and a higher incidence of multivessel coronary disease than patients who have never had CABG. In patients with previous $C A B G$, the infarct related artery (IRA) was split evenly between a bypass graft $(n=50)$ and a native vessel $(n=54)$. Procedural success (defined as TIMI 3 flow at the end of procedure) was less likely in patients with previous CABG $84.5 \%$ vs $95.6 \%$ $(p<0.0001)$ than in patients who had never undergone CABG. Patients with previous CABG had more MACE $(32.8 \%$ vs $16.5 \%$, $\mathrm{p}=0.01$ ) during the 4-year follow-up period (Abstract 130 figure 1). After multivariable adjustment this difference persisted (HR 2.2, $95 \%$ CI 1.26 to $3.78, p=0.02$ ). When stratifying prior CABG patients by the type of IRA (Abstract 130 figure 2); Long term MACE were significant more likely in patients who had bypass graft PCI than in patients that had native vessel PCI (44.6\% vs $19.8 \%, p=0.04)$.

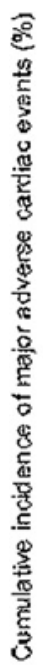

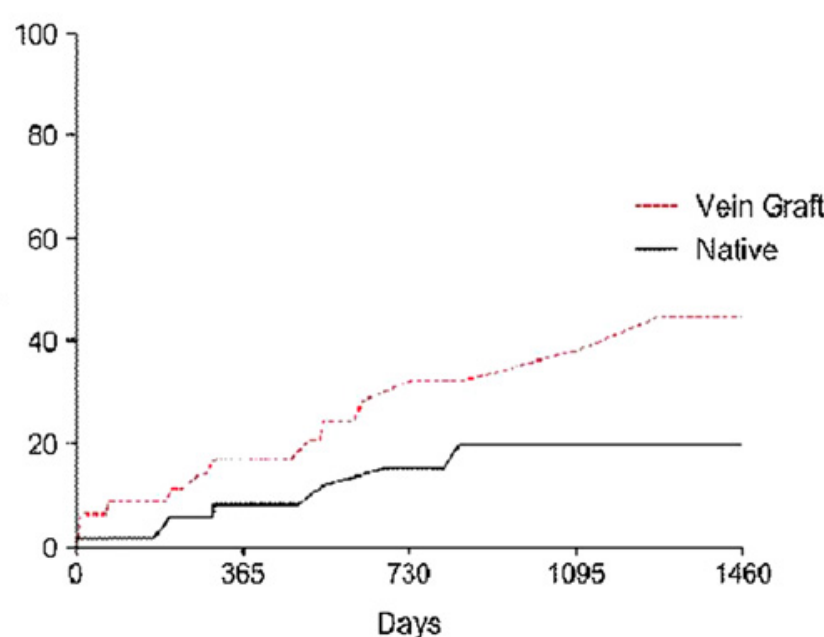

Abstract 130 Figure 2

Conclusions Previous CABG patients with STEMI treated with primary PCI have higher long-term adverse events. The long-term event rates are higher still if the IRA is a bypass graft.

\section{MULTIVESSEL CORONARY DISEASE IN PRIMARY PERCUTANEOUS INTERVENTION. REVASCULARISATION STRATEGY AND ITS IMPACT ON MEDIUM TERM MORTALITY}

doi:10.1136/heartjnl-2012-301877b.131

A E Alahmar,* A S Banning, N S Rajendra, B Wrigley, K Pujara, A H Gershlick. Glenfield Hospital, University Hospitals of Leicester NHS Trust, Leicester, UK

Introduction The optimal revascularisation strategy for patients presenting with ST-Segment Elevation MI (STEMI) and multivessel coronary disease remains under debate. Although current practice in patients without cardiogenic shock is to treat the infarct-related artery only at the time of presentation, it is not clear whether residual coronary disease should be treated either during the acute admission or post-discharge.

Methods All Patients presenting to UHL between April 2008 and November 2011 with STEMI undergoing Primary PCI were analysed retrospectively. The outcome measure was mortality either inhospital or post-discharge. All patients were followed-up until end of November 2011, follow-up duration is expressed as mean \pm SD. Patients were divided into three groups; PCI for single vessel disease (SVD), Multivessel disease (MVD) \& single vessel PCI and MVD \& multivessel PCI. Baseline characteristics (age, gender, risk factors, 
TIMI flow pre- and post-procedure, smoking history, cardiogenic shock, previous MI, CABG or PCI) were compared between groups. Statistical comparison was made using $\chi^{2}$ test for categorical data and $t$ test for continuous data.

Results 829 patients underwent Primary PCI between April 2008 and November 2011. 530 had single vessel disease. 299 patients had MVD; 193 underwent single vessel angioplasty to the infarct related artery, 71 patients underwent multivessel PCI at time of primary PCI and 35 had a further PCI either during that admission or post-discharge. Mean follow-up was $524 \pm 347$ days. Overall in-hospital mortality was $3.5 \%$ and mortality to follow-up was $8.5 \%$. Baseline characteristics were similar between groups with the exception of previous MI and Previous CABG which was significantly higher among patients with MVD \& single vessel PCI. Cardiogenic shock was also higher among patients with MVD \& multivessel PCI (19\% vs SVD 3.8\%, p<0.05). In-hospital mortality between the three groups was similar; $\mathrm{SVD}=3 \%$, MVD\& single vessel $\mathrm{PCI}=4.9 \%$, MVD \& multivessel $\mathrm{PCI}=2.9 \%$, $\mathrm{p}=0.203$. Overall mortality to follow-up between the three groups was also similar (Abstract 131 table 1, $\mathrm{p}=0.20$ ). Exclusion of cardiogenic shock demonstrated a trend towards improved overall mortality in patients undergoing multivessel PCI (Abstract 131 table 2, $p=0.17$ ). Looking exclusively at patients post-discharge, a similar trend towards improved mortality with multivessel PCI was seen (SVD $=5.1 \%$, MVD with single vessel $\mathrm{PCI}=4.97 \%, \mathrm{MVD}$ disease with multivessel $\mathrm{PCI}=1.94 \% ; \mathrm{p}=0.372$ ).

\section{Abstract 131 Table 1}

\begin{tabular}{lllc}
\hline & $\begin{array}{l}\text { Total } \\
\text { number }\end{array}$ & $\begin{array}{l}\text { Number of deaths } \\
\text { to follow-up }\end{array}$ & Mortality (\%) \\
\hline $\begin{array}{l}\text { Single vessel disease } \\
\begin{array}{l}\text { Multivessel disease: } \\
\text { single vessel PCl }\end{array}\end{array}$ & 530 & 49 & 9.2 \\
$\begin{array}{l}\text { Multivessel disease: } \\
\text { multivessel PCl }\end{array}$ & 196 & 20 & 10.4 \\
\hline
\end{tabular}

Abstract 131 Table 2

\begin{tabular}{llll}
\hline & $\begin{array}{l}\text { Total number excl. } \\
\text { cardiogenic shock }\end{array}$ & $\begin{array}{l}\text { Number of deaths } \\
\text { to follow-up }\end{array}$ & Mortality (\%) \\
\hline $\begin{array}{l}\text { Single vessel disease } \\
\begin{array}{l}\text { Multivessel disease: } \\
\text { single vessel PCl }\end{array}\end{array}$ & 509 & 44 & 8.6 \\
$\begin{array}{l}\text { Multivessel disease: } \\
\text { multivessel PCl }\end{array}$ & 97 & 17 & 9.3 \\
\hline
\end{tabular}

Conclusions These findings show a trend towards lower mortality post-discharge with mutlivessel PCI carried out in STEMI patients with MVD, suggesting consideration should be given to complete revascularisation in haemodynamically stable STEMI patients with multivessel disease, either during acute admission or post-discharge. The results of the ongoing UK multicentre randomised trial CVLPRIT will address this important aspect of managing patients with STEMI and multivessel disease.

\section{COMBINED PRASUGREL AND BIVALIRUDIN TREATMENT DURING PRIMARY PCI OFFERS A SAFE AND EFFECTIVE STRATEGY IN ST-ELEVATION MI}

doi:10.1136/heartjnl-2012-301877b.132

T W Johnson, ${ }^{*}$ J W Strange, V Oriolo, J Edmond, A Davies, B C Reeves, A Baumbach. Bristol Heart Institute, Bristol, UK

Introduction Successful treatment of STEMI requires early diagnosis and urgent passivation of the culprit lesion by antithrombotic
Abstract 132 Table 1

\begin{tabular}{llll}
\hline & All patients, $\mathbf{n = 5 3 3}$ & Group A, $\mathbf{n = 3 4 5}$ & Group B, $\mathbf{n = 1 8 8}$ \\
\hline Age (median) & 63.6 & 58.8 & 72.3 \\
Male sex (\%) & 78.8 & 82.9 & 71.3 \\
Diabetes (\%) & 11.1 & 7.8 & 17.0 \\
Hypertension (\%) & 30.6 & 26.1 & 38.8 \\
Hypercholesterolaemia (\%) & 30.6 & 30.1 & 31.4 \\
Current smoking (\%) & 33.2 & 39.4 & 21.8 \\
Previous MI (\%) & 8.6 & 7.5 & 10.6 \\
Previous CABG (\%) & 2.3 & 1.2 & 4.3 \\
Previous PCI (\%) & 6.4 & 5.5 & 8.0 \\
\hline
\end{tabular}

therapy and mechanical revascularisation. A delicate balance exists between the risk of thrombosis and bleeding, consequently the choice of antithrombotic therapy is critical. Bivalirudin, a direct thrombin inhibitor, has been shown to offer a superior safety profile over heparin and GP2b3a inhibition in STEMI (Horizons-AMI). Similarly, the novel P2Y12-receptor inhibitor, Prasugrel, achieves faster and more consistent platelet inhibition than clopidogrel with improved clinical outcomes in STEMI (TRITON-TIMI 38). Although untested in a randomised clinical trial, the combination of bivalirudin and prasugrel for the treatment of STEMI appears to offer fast and effective inhibition of thrombosis with an acceptable bleeding profile. A randomised trial is in progress (BRAVE 4) but data will not be available until 2013.

Methods Since June 2010 our preferred treatment strategy for patients presenting with STEMI has been pre-loading with prasugrel $60 \mathrm{mg}$ and peri-procedural bivalirudin $(0.75 \mathrm{mg} / \mathrm{kg}$ bolus followed by an infusion of $1.75 \mathrm{mg} / \mathrm{kg} / \mathrm{h}$ ). Patients $>75 \mathrm{yrs},<65 \mathrm{~kg}$, with a history of cerebrovascular accident, pre-treated with clopidogrel or intubated without NG tube access were excluded and received treatment according to operator preference. The preferred access site was the radial artery and all operators were experienced in performing percutaneous coronary intervention from this route. Patients were discharged on lifelong aspirin and prasugrel $10 \mathrm{mg}$ for 12 months. Additionally, patients routinely received statin, ACE inhibitor and $\beta$-blocker therapy. Consecutive patients were enrolled over a 12-month period commencing in July 2010

Results In total 533 patients presented with STEMI and underwent treatment with PPCI in the study period. Of these 345 received a prasugrel loading dose and bivalirudin treatment (Group A). The remaining 188 patients received alternative therapy (Group B). Baseline demographics (Abstract 132 table 1) differ significantly between groups due to the criteria used for drug regimen selection. Procedural outcomes are summarised in Abstract 132 table 2. Procedural success was achieved in $97.6 \%$ of patients, using radial access in $79.9 \%$. Bleeding occurred in $1.9 \%$ of all patients (Group A $1.2 \%)$. Stent thrombosis occurred in $1.1 \%$ of patients and no definite

Abstract 132 Table 2

\begin{tabular}{llll}
\hline & All patients $\mathbf{n = 5 3 3}$ & Group A $\mathbf{~}=\mathbf{3 4 5}$ & Group $\mathbf{B} \mathbf{~ n = 1 8 8}$ \\
\hline All Bleeding (\%) & 1.9 & 1.2 & 3.2 \\
Stent Thrombosis (\%) & 1.1 & 0.9 & 1.6 \\
Definite ST (\%) & 0.6 & 0.6 & 0.5 \\
Probable ST (\%) & 0.6 & 0.3 & 1 \\
Reinfarction (\%) & 0.6 & 0.6 & 0.5 \\
Reintervention (\%) & 3.9 & 3.8 & 4.3 \\
Stroke (\%) & 0.8 & 0.3 & 1.6 \\
In-hospital mortality (\%) & 5.3 & 2.6 & 10.1 \\
30-day mortality (\%) & 6 & 2.6 & 12.2 \\
\hline
\end{tabular}

